\title{
Analisis Exploratorio En La Industria De Quesos Artesanales En Escárcega, Campeche De Acuerdo A Los Prerrequisitos De Haccp
}

\author{
Maximiliano Vanoye Eligio (Dr. En Manejo de Recursos \\ Naturales Tropicales) \\ Jorge Isidro Xool Alfaro (Ing. Agronomía) \\ José Ángel de Espinoza López (Ingeniero en Industrias \\ Alimentarias) \\ Jorge Emmanuel Arteaga Castillo (Lic. En Administración) \\ Instituto Tecnológico Superior de Escárcega \\ Ingeniería en Industrias Alimentarias. Calle $85 \mathrm{~s} / \mathrm{n}$ entre 10B, Col. Unidad \\ Esfuerzo y Trabajo I; Tel: 982 5960603; Tel: 9825960604.
}

doi: 10.19044/esj.2017.v13n18p592 URL:http://dx.doi.org/10.19044/esj.2017.v13n18p592

Abstract

This article presents a diagnosis of hygiene management based on the fulfillment of the prerequisites of a HACCP system, in three companies of the agrifood sector that operate in the municipality of Escárcega, Campeche, especially in the elaboration of artisanal cheeses. The evaluation methodology corresponded to the verification of compliance with the checklist of the instrument GHYCAL (Hygiene and Quality Management). The results allowed to find levels of hygiene management in a percentage scale and to classify the three companies in low level of compliance for all, due mainly to the little knowledge and technological development that is presented in the region.

Keywords: Artisanal cheesemakers, GHYCAL instrument, Escárcega

Resumen

En este artículo se presenta un diagnóstico de la gestión de la higiene basado en el cumplimiento de los prerrequisitos de un sistema HACCP, en tres empresas del sector agroalimentario que operan en el municipio de Escárcega, Campeche, en especial en la elaboración de quesos artesanales. La metodología de evaluación correspondió a la verificación del cumplimiento de la lista de chequeo del instrumento GHYCAL (Gestión de la Higiene y Calidad). Los resultados permitieron encontrar niveles de gestión de la higiene en una escala porcentual y clasificar a las tres empresas 
en nivel de cumplimiento bajo para todas, esto es debido principal al poco conocimiento y desarrollo tecnológico que se presenta en la región.

Palabras Clave: Queserías artesanales, instrumento GHYCAL, Escárcega

\section{Introduction}

La producción de ganado con doble propósito que se presenta en México tiene un gran impacto, ya que en áreas tropicales del país abarca 51.3 millones de hectáreas, equivalentes al $26.2 \%$ del territorio nacional (Durango, Zacatecas, Coahuila, San Luis, Potosí, Guanajuato, Tamaulipas, Puebla, Veracruz, Tabasco, Yucatán, Campeche, Quintana Roo, Chiapas, Oaxaca, Nayarit, Jalisco, Hidalgo, Colima, Guerrero y Morelos) (Herrera y Saldaña, 1999), por otra parte, la producción de leche y carne que actualmente se produce en el país es insuficiente para satisfacer la demanda debido al incremento demográfico y la urbanización, motivo por el cual el trópico mexicano representa una gran alternativa para incrementar su participación en la producción nacional (INEGI, 2001). Esta condición que se presenta en México en el área de la ganadería productora de leche permite que se considere como prioritaria dentro de los programas de fomento y un punto fundamental en la orientación de políticas públicas que tienen por objeto incentivar su producción, para transformarla en una actividad altamente productiva y competitiva (Villamar et al., 2004). Además, a la leche se le considera como uno de los alimentos más completos, debido a su contenido de nutrientes entre los que destacan las proteínas, caracterizadas por su gran contenido de aminoácidos esenciales, razón por la cual es considerada mundialmente como un alimento ideal y necesario (Ares, 1994). Por otro lado, la leche es uno de los alimentos perecederos y que requiere mucha manipulación. En el 2000 se registraron más de 200 casos de intoxicación alimentaria tan sólo en un país africano, donde los alimentos involucrados fueron productos lácteos vendidos en la calle, ya que la calidad microbiológica de los alimentos que se venden en las calles no presenta inocuidad, por el cual se ha investigado en diferentes ciudades y países para demostrar el riesgo epidemiológico que representan. Esto ha repercutido en el sector lácteo, ya que han consolidado el establecimiento de las agroindustrias del sector lácteo con incremento en la escala, concentración e inocuidad, así como la modificación en el patrón de demanda de los consumidores por alimentos procesados, en detrimento de productos básicos (Reardon y Barrett, 2000; Dirven, 2001), mientras que la tendencia local ha consistido en estimular actividades con mayor valor agregado que repercutan en el aumento de la producción agropecuaria y en el nivel de vida de las poblaciones más afectadas, como las rurales (Castañeda et al., 2009). En los años recientes, la agroindustria de lácteos en México fue la más dinámica 
dentro del sector agroalimentario; participó con $10 \%$ del valor total del sector y contribuyó con $0.6 \%$ al Producto Interno Bruto (PIB) y contó con 12,000 establecimientos que generaron 66,000 empleos (Aguilar, 2003). La agroindustria de lácteos en México está conformada por el conjunto de empresas involucradas en la transformación de leche y derivados. Dentro de su estructura coexisten grandes empresas en número reducido, con un gran número de pequeñas agroindustrias con problemas para articularse al mercado y problemas de calidad de sus productos (Espinosa et al., 2006).

Por otra parte, la industrialización de la leche en el país se efectúa en relación a cuatro segmentos de especialización: tratamiento y envasado de leche fluida, leche industrializada (condensada, evaporada y en polvo), elaboración de yogurt y la fabricación de quesos, cuya participación para inicios de esta década fue de $11 \%$ en la producción nacional de productos lácteos (FIRA, 2001). En el caso de las pequeñas empresas responsables de elaboración de quesos artesanales (QA) presenta una condición particular. La producción está concentrada por las grandes agroindustrias transnacionales y nacionales (Montañez et al., 2006). Las pequeñas empresas tienen una participación activa, no contabilizada ni caracterizada, y su contribución a la producción se estima por la diferencia entre el total producido a nivel nacional y lo aportado por las empresas de mayor escala. La leche procesada en las queserías artesanales de Escárcega proviene de una región productiva ganadera de la parte sur del estado de Campeche, conocida como "La región de los Ríos" en la cual están incluidos los municipios de Escárcega, Candelaria, Palizada y Ciudad del Carmen. Sin embargo, es indudable que las condiciones de suelo y clima de esta región son las ideales para la producción de leche y sus derivados, tales como los Quesos Artesanales en un excelente negocio. La contribución familiar en la elaboración de quesos artesanales son las bases del desarrollo sostenido de estos establecimientos, los cuales atienden el mercado interno. Es por ello, que el principal objetivo de este trabajo de investigación fue evaluar la situación actual que presentan las queserías en el municipio de Escárcega, Campeche en base a los prerrequisitos de HACCP.

\section{Materiales y métodos:}

El estudio se realizó en la ciudad de Escárcega, la cual forma parte del municipio de Escárcega, Campeche, México. El municipio de Escárcega se ubica geográficamente entre los paralelos $18^{\circ} 51^{\prime}$ y $18^{\circ} 09^{\prime}$ de latitud Norte y los paralelos 90'20' y 91o 33' de longitud Oeste (INEGI, 2006). Tiene una altura de 60 metros sobre el nivel del mar y una extensión de 4,569.64 km2, que corresponden $8.04 \%$ de la superficie total de la entidad (Fig. 1). En este municipio la elaboración de quesos artesanales es una práctica común, sin embargo, tres empresas son las dominantes del mercado 
de la comercialización de quesos artesanales, las cuales fueron elegidas para su estudio.

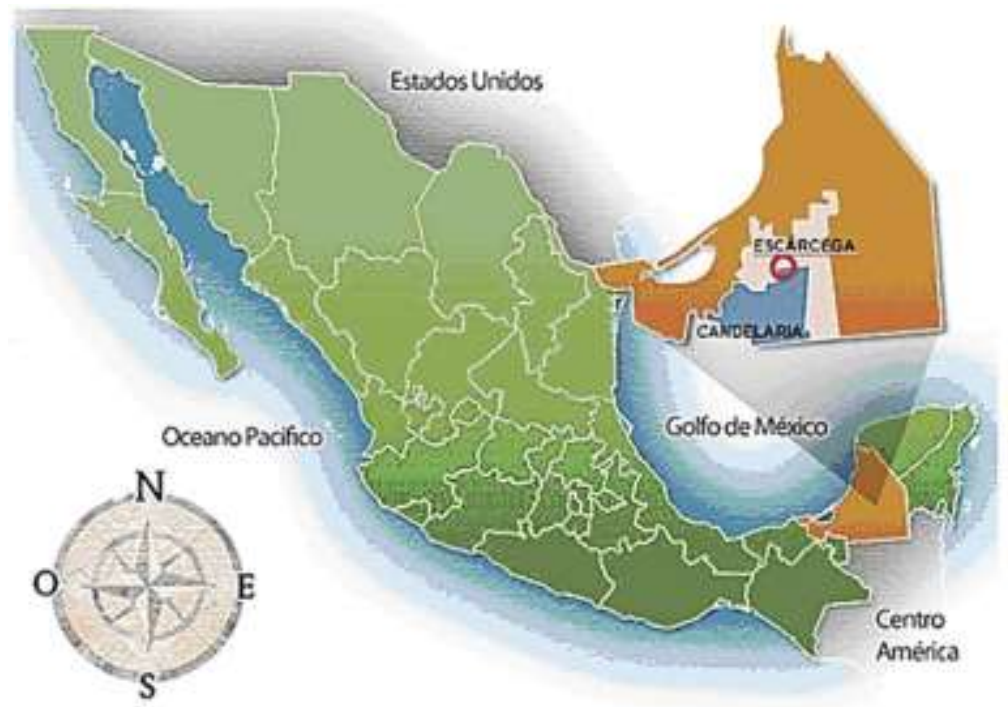

Fig. 1. Ubicación geográfica del municipio de Escárcega, Campeche, México (DOF, 2014).

La metodología que se utilizó en este estudio de investigación se hizo de acuerdo a Van Der Spiegel (2005) menciona que el diseño de instrumentos de evaluación requiere de un procedimiento ordenado asociado con el rigor científico que le confiere ciertas características de estructura, contabilidad, validez y objetividad; de manera general, la metodología utilizada para la construcción del instrumento GHYCAL, corresponde a la metodología utilizada en la construcción de instrumentos para evaluar la gestión de la calidad en la industria alimentaria, que incluye un extenso proceso de revisión de literatura para la formulación del instrumento preliminar, validación de contenido utilizando sesiones Delphi, análisis de contabilidad interna y validación de criterio. En primer lugar se definió la variable a medir que en este caso correspondió a "Gestión de la higiene", con unidades porcentuales para poder presentar los resultados en función de los niveles de cumplimiento de cada empresa evaluada. Seguidamente se definió la forma del instrumento, que correspondió a una estructura basada en sets de criterios, por lo que el instrumento presentó 7 conjuntos de criterios, uno por cada uno de los siete planes previos de higiene y trazabilidad en un sistema HACCP; posteriormente y después de un extenso proceso de revisión bibliográfica, se definieron 51 ítems que corresponden a aquellos aspectos enmarcados en los siete planes de higiene y trazabilidad que definen el programa de prerrequisitos de un sistema HACCP (Cuadro 1). 
Cuadro 1. Items de planes de higiene y trazabilidad para evaluar en queserías escarceguenses.

\section{Instrumento GHYCAL (Gestión de la higiene y calidad en alimentos)-} VALORACIÓN DEL NIVEL DE GESTIÓN DE LA HIGIENE

\section{PLAN DE CONTROL DE AGUAS}

1.1 ¿Actualmente la empresa cuenta con una fuente de abastecimiento suficiente de agua potable?

1.2 ¿La empresa cuenta con un deposito suficiente de almacenamiento de agua potable, diseñado y construido con materiales sugeridos por las autoridades sanitarias y que además permitan realizar los procesos como mínimo por una jornada?

1.3 ¿Realiza con la periodicidad adecuada análisis de laboratorio para verificar la potabilidad del agua de uso en la empresa?

1.4 ¿Los análisis que se realizan en la empresa incluyen: cloro residual, parámetros físico-químicos y microbiológicos establecidos en la legislación?

1.5 ¿La empresa cuenta con un plan documentado de acciones correctivas que le permita actuar rápidamente en caso de encontrar niveles de potabilidad inadecuados en el agua?

1.6 ¿Cuenta con un documento que les permita conocer y consultar la normatividad nacional en cuanto a los niveles permisibles de cada uno de los parámetros que determinan la calidad del agua potable?

\section{PLAN DE LIMPIEZA Y DESINFECCIÓN}

2.1 ¿La empresa cuenta con un programa escrito que especifique las operaciones de limpieza y desinfección de todos los equipos, instalaciones, utensilios, accesorios y vehículos, así como la periodicidad con que han realizarse y el personal responsable de llevarlas a cabo?

2.2 ¿Las operaciones de limpieza y desinfección implementadas, tienen en cuenta las características de cada una de las zonas de la empresa, la naturaleza de la suciedad, la contaminación y tipo de superficie en función de su contacto no con los alimentos procesados?

2.3 ¿L a empresa cuenta con un listado, descripción y manual de manejo de los productos que se utilizan para la limpieza y desinfección y dispone de las fichas técnicas que garanticen que estos estén autorizados para su uso en la industria alimentaria?

\section{4 ¿La empresa cuenta con los utensilios suficientes y adecuados para la ejecución} de las labores de limpieza y desinfección y estos son limpiados, revisados y sustituidos con la frecuencia adecuada?

2.5 ¿L a empresa cuenta con procedimientos documentados para comprobar la eficacia de los procesos de limpieza y desinfección?

2.6 ¿Las actividades de limpieza y desinfección incluyen todas las partes de difícil acceso de las máquinas y equipos?

2.7 ¿Cuenta la empresa con registro donde se indique la hora y fecha de realización de las labores de limpieza y desinfección y con la firma de los responsables?

\begin{tabular}{|l|l|l|l|l|}
1 & 2 & 3 & 4 & 5 \\
1 & 2 & 3 & 4 & 5 \\
1 & 2 & 3 & 4 & 5 \\
1 & 2 & 3 & 4 & 5 \\
1 & 2 & 3 & 4 & 5 \\
1 & 2 & 3 & 4 & 5 \\
\hline
\end{tabular}

\begin{tabular}{|l|l|l|l|l|l|}
\hline $\mathrm{y}$ & 1 & 2 & 3 & 4 & 5 \\
\hline 1 & 2 & 3 & 4 & 5 \\
\hline os & 1 & 2 & 3 & 4 & 5 \\
\hline 1 & 2 & 3 & 4 & 5 \\
\hline 1 & 2 & 3 & 4 & 5 \\
\hline 1 & 2 & 3 & 4 & 5 \\
\hline
\end{tabular}


2.8 iAl hacer una inspección visual en pisos, paredes, puertas, ventanas, techos, mesas de trabajo y equipos, se observa presencia de materia extraña o evidencia de suciedad?

\section{PLAN DE CONTROL Y FORMACIÓN DE MANIPULADORES DE LOS ALIMENTOS}

3.1 ¿L a totalidad de los empleados de la empresa, que tienen contacto con los alimentos, poseen el respectivo conocimiento de manipulador de alimentos?

$3.2 ¿$ El personal que tiene contacto con alimento, ha recibido capacitación basada en buenas prácticas de manipulación e higiene de alimentos?

3.3 ¿La empresa cuenta con un programa escrito que defina las actividades de capacitación para los empleados que manipulan alimentos?

3.4 ¿Realizan algún tipo de inducción para los empleados nuevos, en temas relacionados con limpieza y buenas prácticas de manipulación e higiene de alimentos?

3.5 ¿Cuenta todo el personal en contacto con alimentos, con la indumentaria adecuada incluyendo tapabocas, gorro, ropa y calzado?

3.6 ¿Existe un plan documentado para la recolección, almacenamiento y eliminación de residuos propios del proceso y está definido un responsable para esta función?

3.7 ¿Los manipuladores cumplen con las normas de higiene en cuanto a actitud, hábitos, comportamientos e instrucciones de trabajo establecidas por la empresa?

3.8 ¿El personal tiene conocimiento de las obligaciones de notificar al responsable de la empresa los síntomas o el padecimiento de enfermedades de transmisión por vía alimentaria y que puedan causar contaminación en los alimentos que se procesan?

3.9 ¿Existe un procedimiento documentado que permita retirar y reemplazar en línea de producción a un operario enfermo que ponga en riesgo la seguridad de los alimentos?

3.10 ¿Cuenta con registro de las actividades de formación en higiene y buenas prácticas de manipulación de alimentos?

3.11 ¿La empresa cuenta con lavamanos y sanitarios suficientes y adecuados, a disposición de los operadores?

3.12 ¿Existen áreas destinadas para la alimentación o descanso de los trabajadores y se encuentran perfectamente definidas y aisladas de las áreas de producción?

3.13 ¿Se realizan exámenes y controles médicos a los trabajadores, tanto al ingreso al empleo como de manera periódica una vez en ejercicios de sus funciones?

\begin{tabular}{|l|l|l|l|l}
1 & 2 & 3 & 4 & 5 \\
1 & 2 & 3 & 4 & 5 \\
1 & 2 & 3 & 4 & 5
\end{tabular}

\begin{tabular}{|l|l|l|l|l|}
\hline 1 & 2 & 3 & 4 & 5 \\
\hline 1 & 2 & 3 & 4 & 5
\end{tabular}

1

1

\begin{tabular}{l|l|l|l}
2 & 3 & 4 & 5
\end{tabular}

\begin{tabular}{|l|}
1 \\
1
\end{tabular}

\begin{tabular}{|l|l|l|l|l|}
1 & 2 & 3 & 4 & 5 \\
\hline 1 & 2 & 3 & 4 & 5
\end{tabular}

\section{PLAN DE MANTENIMIENTO PREVENTIVO}

4.1 ¿L a empresa dispone de un documento escrito de inspección periódica para comprobar el estado de las instalaciones y equipo?

4.2 ¿El estado actual de los equipos e instalaciones, permiten operar en condiciones adecuadas de acuerdo a los requerimientos del sector agroalimentario? 4.3 ¿Dispone de un programa de calibración y verificación de equipos e instrumentos de medidas?

\begin{tabular}{|l|l|l|l|l|l|}
\hline os & 1 & 2 & 3 & 4 & 5 \\
\hline 1 & 2 & 3 & 4 & 5 \\
\hline 1 & 2 & 3 & 4 & 5 \\
\hline 1 & 2 & 3 & 4 & 5 \\
\hline 1 & 2 & 3 & 4 & 5 \\
\hline & 1 & 2 & 3 & 4 & 5 \\
\hline & 1 & 2 & 3 & 4 & 5 \\
\hline 1 & 2 & 3 & 4 & 5 \\
\hline
\end{tabular}


4.4 ¿Cuentan con registros que soporten el cumplimiento de los procesos de calibración y verificación de los equipos de medidas?

4.5 ¿Poseen personal calificado para las operaciones de mantenimiento o contratan la prestación de estos servicios con firmas especializadas que pueden certificar su idoneidad?

4.6 ¿Las labores de mantenimiento son realizadas periódicamente de acuerdo a una programación establecida que incluye todas las áreas de producción?

4.7 ¿Cuenta la empresa con un plan documentado de acciones correctivas para solucionar eventualidades cuando se detecte un fallo en el funcionamiento de un equipo?

\section{PLAN DE CONTROL DE PLAGAS Y SISTEMA DE VIGILANCIA}

5.1 ¿Dispone la empresa de algún plan documentado para el control y prevención de plagas?

5.2 ¿El personal que realiza las labores de control de plagas, cuenta con el conocimiento de aplicador de productos fitosanitarios?

5.3 ¿Existen planos de localización de todas las estaciones de control de plagas y se encuentran correctamente identificados los elementos utilizados para el control y prevención de la presencia de plaga?

5.4 ¿Existe registro de aplicación que incluyan tipo y dosis de plaguicidas utilizados, plazos de seguridad, personal responsable de la aplicación y que este fechado y debidamente firmado?

5.5 ¿Los productos utilizados para el control de plagas, están debidamente etiquetados y su uso está autorizado para la industria alimentaria?

5.6 ¿La industria efectúa acciones de vigilancias para comprobar la efectividad del plan y así mismo la ausencia o erradicación de las plagas combatidas?

5.7 ¿En caso que el plan de control de plagas resulte ineficiente, cuenta con un plan documentado de acciones correctivas que incluyan medidas oportunas diferentes a los tratamientos que se han empleado con anterioridad?

\section{PLAN DE CONTROL DE TRAZABILIDAD}

6.1 ¿La empresa un listado detallado de los proveedores encargados de suministrar las materias primas y materiales auxiliares?

6.2 ¿La empresa tiene establecidos los mínimos, higiénicos, sanitarios y de calidad, que exige a sus proveedores para garantizar la inocuidad de cada una de las materias primas?

6.3 ¿La empresa cuenta con especificaciones precisas de las características para la adquisición de cada una de las materias primas?

6.4 ¿Cuenta con personal encargado de verificar e inspeccionar que las materias primas cumplan las especificaciones establecidas por la empresa?

6.5 ¿Se registra suficiente información en el momento de la recepción, de acuerdo a las establecidas, para aceptar o rechazar el ingreso de materias primas?

6.6 ¿Existe programas establecidos para la destrucción o devolución de materias primas rechazadas?

6.7 ¿Se dispone de registros de control de producción que relacionen la materia prima y el número de lotes de los productos elaborados o manipulados cada día?

\begin{tabular}{|l|l|l|l|l}
1 & 2 & 3 & 4 & 5 \\
1 & 2 & 3 & 4 & 5 \\
1 & 2 & 3 & 4 & 5 \\
1 & 2 & 3 & 4 & 5 \\
\hline
\end{tabular}

\begin{tabular}{|l|l|l|l|l|}
\hline 1 & 2 & 3 & 4 & 5 \\
\hline 1 & 2 & 3 & 4 & 5 \\
\hline 1 & 2 & 3 & 4 & 5 \\
\hline 1 & 2 & 3 & 4 & 5 \\
\hline 1 & 2 & 3 & 4 & 5 \\
\hline 1 & 2 & 3 & 4 & 5 \\
\hline
\end{tabular}

\begin{tabular}{|l|l|l|l|l|l|}
\hline & 1 & 2 & 3 & 4 & 5 \\
\hline 1 & 2 & 3 & 4 & 5 \\
\hline 1 & 2 & 3 & 4 & 5 \\
\hline 1 & 2 & 3 & 4 & 5 \\
\hline 1 & 2 & 3 & 4 & 5 \\
\hline 1 & 2 & 3 & 4 & 5 \\
\hline
\end{tabular}


6.8 ¿La empresa cuenta con la capacidad y el personal necesario para identificar y retirar el producto del mercado y de los canales de distribución en caso necesario? 6.9 ¿Al registrarse alguna queja o reclamo referido a la calidad o inocuidad del producto y al ser valorado por la empresa, es posible establecer la causa del deterioro del producto?

6.10 ¿Existe un procedimiento documentado para el retiro o cuarentena en caso de que se detecten anomalías o contaminación de los productos en el interior de la empresa?

\begin{tabular}{|l|l|l|l|l|l|}
1 & 2 & 3 & 4 & 5 \\
\hline 1 & 1 & 2 & 3 & 4 & 5 \\
\hline
\end{tabular}

La definición de la escala de valoración es tal vez uno de los aspectos considerados clave dentro del proceso de construcción de cualquier instrumento de evaluación, debido a que por un lado se debe conseguir una escala de fácil interpretación por los evaluadores y los evaluados, pero por otro lado se debe de seleccionar una escala que permita medir la variable en toda su extensión, puesto que cuantos más valores sean permitidos a la variable, mayor provecho puede ser obtenido en la evaluación del instrumento, porque podrá determinarse con mayor exactitud la varianza entre las diferentes respuestas obtenidas de diferentes empresas evaluadas. La escala seleccionada para el instrumento GHYCAL, correspondió a una escala discreta de cinco categorías (Cuadro 2) que durante el proceso de evaluación de cada empresa (queserías), deben ser asignadas según el nivel de cumplimiento de cada criterio evaluado, ponderando la información suministrada por la empresa y el resultado de una inspección visual realizada en la planta de producción de alimentos.

Cuadro 2. Escalas de medición para el instrumento GHYCAL

\begin{tabular}{|c|c|c|}
\hline CATEGORIA & $\begin{array}{c}\text { NIVEL DE } \\
\text { CUMPLIMIENTO }\end{array}$ & DESCRIPCIÓN \\
\hline 1 & No hay cumplimiento & $\begin{array}{l}\text { Cuando no existen las condiciones } \\
\text { mínimas requeridas en la ejecución de } \\
\text { una acción. }\end{array}$ \\
\hline 2 & Mínimo cumplimiento & $\begin{array}{lll}\text { Cuando se } & \text { presenta una } & \text { intención } \\
\text { verificable } & \text { encaminada } \\
\text { hacía la } \\
\text { ejecución de una acción. }\end{array}$ \\
\hline 3 & Mediano cumplimiento & $\begin{array}{l}\text { Cuando las condiciones de operación } \\
\text { de una acción o plan se encuentran } \\
\text { medianamente elaboradas con algunos } \\
\text { procedimiento establecidos, pero se } \\
\text { omiten algunos parámetros. }\end{array}$ \\
\hline 4 & $\begin{array}{l}\text { Nivel importante de } \\
\text { cumplimiento }\end{array}$ & $\begin{array}{l}\text { Cuando los requerimientos cuentan } \\
\text { con procedimientos establecidos, pero } \\
\text { se omiten algunos parámetros o no se } \\
\text { exige su cumplimiento. }\end{array}$ \\
\hline 5 & Cumplimiento óptimo & $\begin{array}{l}\text { Cuando se cumple satisfactoriamente } \\
\text { los procedimientos establecidos y se } \\
\text { verifica su cumplimiento }\end{array}$ \\
\hline
\end{tabular}




\section{Resultados y discusión}

De acuerdo a los resultados obtenidos en tres empresas responsables de la elaboración de quesos artesanales y reconocidos en el municipio y en el estado (fig. 2, 3, 4, 5, 6, 7) se puede detectar que el perfil de cumplimiento de las tres empresas evaluadas con el instrumento utilizado (GHYCAL); la puntuación obtenida por cada empresa corresponde al valor porcentual resultante de relacionar el total de puntos obtenidos con respecto al total de puntos que podría haber obtenido si todas los ítems hubiesen sido valorados con cumplimiento óptimo. Como puede verse, en las tres empresas evaluadas, los resultados son muy similares (niveles bajos en gestión de la calidad), indicando una alta correlación entre los resultados obtenidos entre las empresas. En otras investigaciones similares respecto a niveles de cumplimiento altos, se pudo establecer que en el sector de lácteos resultaron 4 empresas con porcentajes superiores (niveles altos de cumplimiento) en relación a la gestión de la higiene.

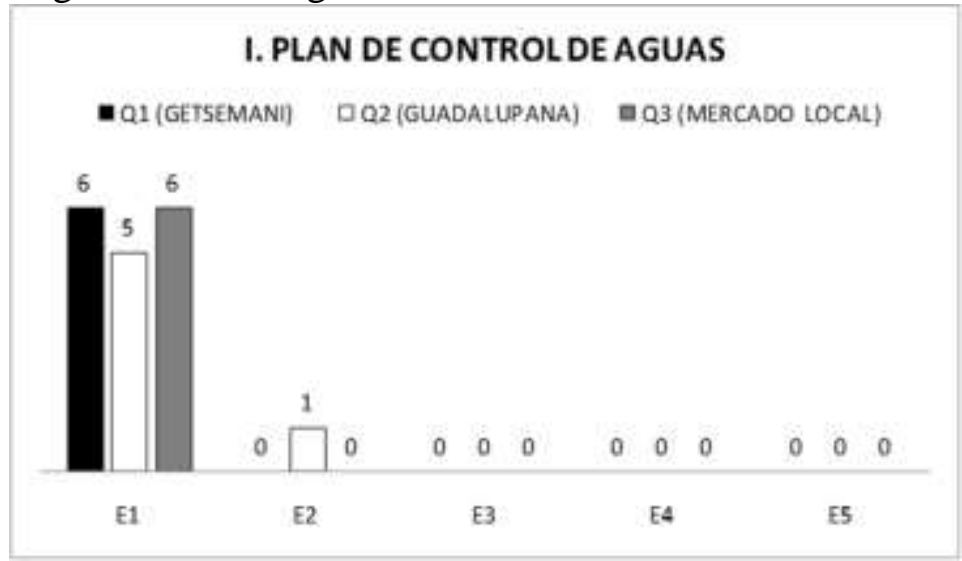

Figura 1. En la figura se puede observar que no existe un control de aguas para la elaboración de quesos en las tres empresas evaluadas.

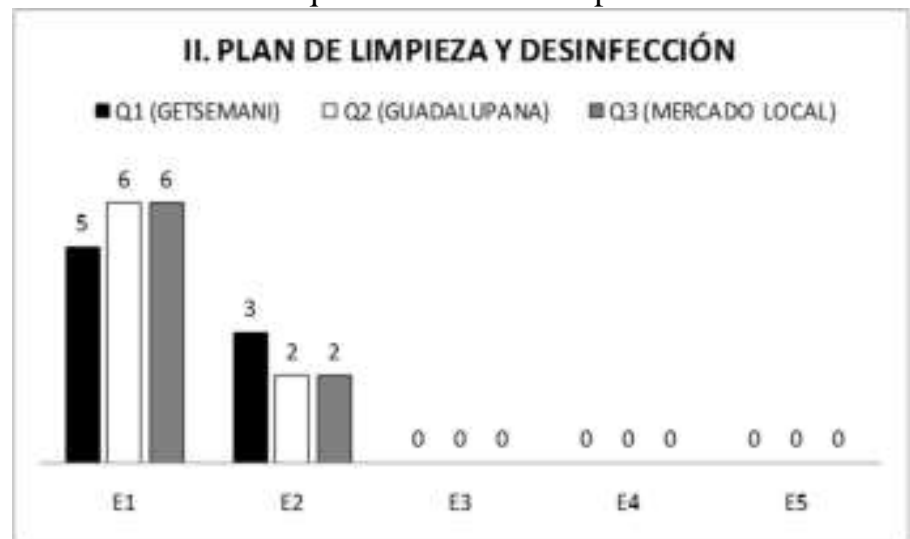

Figura 2. Se puede apreciar que las tres empresas no cuentan con un plan de limpieza y desinfección de los utensilios y maquinaria utilizada en la elaboración de quesos. 


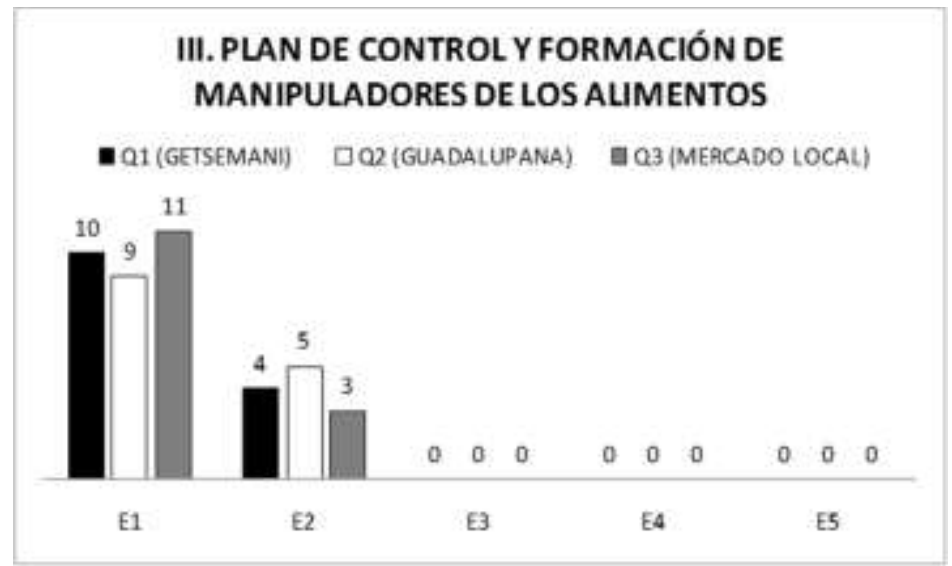

Figura 3. Respecto a la manipulación de los alimentos por personal capacitado, se puede observar que en algunas empresas se tiene la intención de elaborar los alimentos de una forma adecuada.

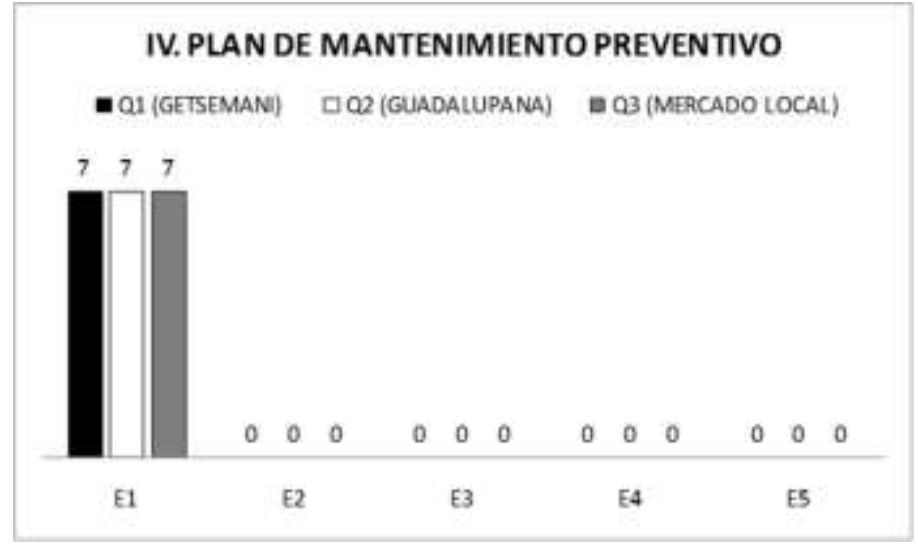

Figura 4. Claramente se observa que en ninguna empresa evaluada se cuenta con un mantenimiento preventivo, lo cual es de suma importancia en cualquier empresa encargada de elaborar alimentos para consumo humano.

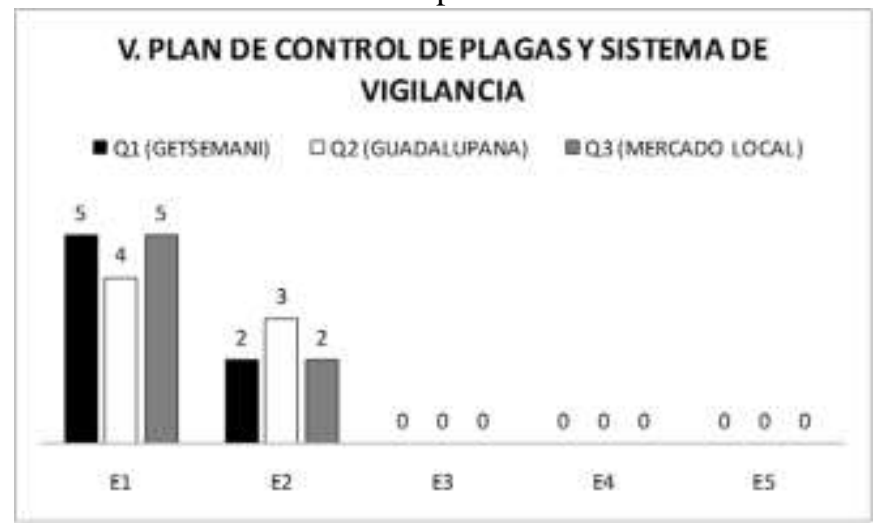

Figura 5. Lo que se puede ver en la gráfica es que las empresas realizan el control de plagas de una manera tradicional con productos que se utilizan en cualquier vivienda, sin embargo no es suficiente, ya que no son los productos adecuados y ni el personal se encuentra capacitado 


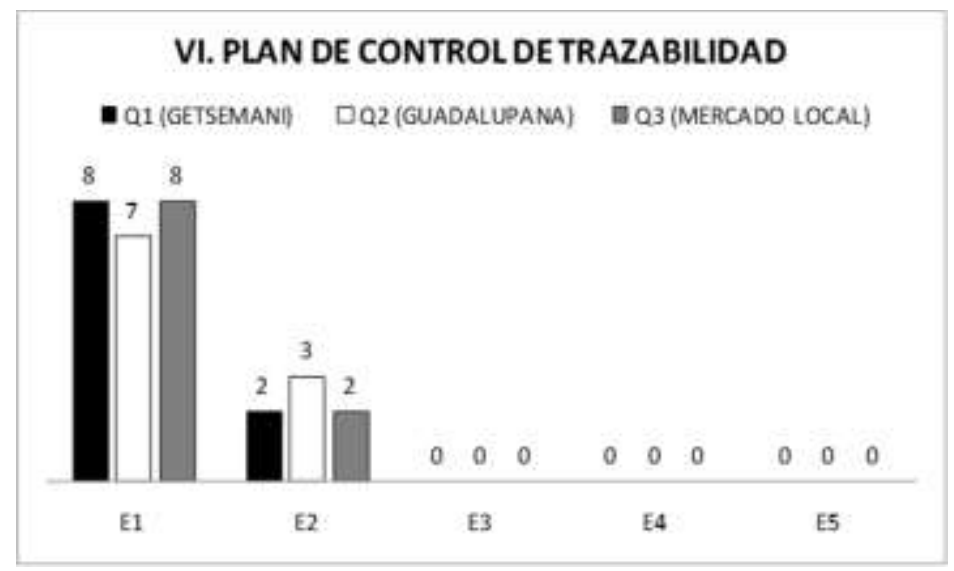

Figura 6. En los que se refiere al control de trazabilidad que deben de llevar las empresas, se puede observar que tienen intentos de llevarlos a cabo, pero aún falta mucho por registrarlo adecuadamente.

\section{Conclusión}

Las empresas evaluadas en el municipio de Escárcega (3) no cuentan con las herramientas necesarias para elaborar productos (quesos), por lo que es necesario implementar cursos con las temáticas que se están evaluado, tal es el caso del curso análisis de peligros y puntos críticos de control de alimentos, El sistema HACCP, el cual es impartido por diversas instituciones públicas y privadas, nacionales e internacionales a las empresas del ramo alimenticio, esto contribuiría a una mejor calidad e higiene de los productos alimenticios.

\section{References:}

1. Aguilar, C. (2003). Tipología de las cadenas industriales y la calidad de la leche en los Altos de Jalisco. Temas de Ciencia y Tecnología. Vol. 7 (19): 13-23.

2. Ares, J. L. (1994). Quesos artesanales: Situación del sector en Andalucía. Agricultura: Revista agropecuaria 743: 474-482.

3. Castañeda, M.T., Boucher, F., Sánchez, V. E. y Espinoza, O. A. (2009). La concentración de agroindustrias rurales de producción de quesos en el noroeste del Estado de México: un estudio de caracterización. Estudios Sociales (Hermosillo, Son.), 17 (34), 73 109.

4. Dirven, M. (2001). Dairy Clusters in Latin America in the Context of Globalization. International Food and Agrobusiness Management. 2 (3/4): 301-313. 
5. DOF, Diario Oficial de la Federación. (2014). http://www.dof.gob.mx/nota_detalle.php?codigo $=5375510 \&$ fecha $=1$ $1 / 12 / 2014$.

6. Espinosa, T., Villegas, A., Gómez, G., Cruz, J. G. y Hernández, A. (2006). La agroindustria láctea en el Valle de México: un ensayo de categorización. Técnica Pecuaria. 44 (2): 181-192.

7. FIRA - Fideicomisos Instituidos en Relación con la Agricultura. (2001). Tendencias y oportunidades de desarrollo de la red leche en México. Boletín Informativo. Vol. 33: 137-15.

8. Herrera y Saldaña R. (1999). La importancia de los maíces y sorgos mejorados para la producción de ensilajes. In: Nuñez G. (coord.). Componentes tecnológicos para producción de ensilados de maíz y sorgo. SAGAR-INIFAP-C.E. La Laguna.

9. INEGI, Instituto Nacional de Estadística Geografía e Informática. (2001). El Sector Alimentario en México. Instituto Nacional de Estadística, Geografía e informática. p. 58.

10. INEGI, Instituto Nacional de Estadística Geografía e Informática. (2006). Monografías del estado de Campeche. http://www.inegi.gob.mx.

11. Montañez, C. D. A., Lozano, M. S. R., Espinosa, F. N. y Morales, R. A. A. (2006). Quesos frescos y semimadurados que se expenden en vía pública en la ciudad de México. Veterinaria Mexicana, 37, 4.

12. Reardon, T. y Barrett, C. (2000). Agroindustrialization, Globalization and International Development, an Overview of Issues, Patterns and Determinants. Agricultural Economics. Vol. 23: 195-205.

13. Van Der Spiegel, M. (2005). Development of the instrument IMAQE-Food to measure effectiveness of quality management. En: International Journal of Quality \& Rellability Management. Vol. 22 (3): 234-255.

14. Villamar, A. L., Pérez, F. H. y Olivera, C. E. (2004). Situación Actual de la Producción de Leche de Bovino en México 2004. Claridades Agropecuarias. No. 136. 